\title{
Estratégias do desporto universitário: um estudo de caso sobre o desporto em universidades portuguesas
}

\author{
Carlos P. Colaço \\ Leandro A. Fleck
}

Faculdade de Motricidade Humana

Universidade Técnica de Lisboa

Portugal

\section{RESUMO}

Este estudo teve como foco principal, aprofundar os conhecimentos sobre o desporto universitário em Universidades

Portuguesas, bem como verificar e compreender as estratégias utilizadas, o planeamento das estratégias, os objectivos e estrutura organizacional dos serviços desportivos. Para tal pesquisa foi utilizado o paradigma qualitativo em estudo de caso, sendo uma metodologia interpretativa, descritiva e fenomenológica. Para a colecta de dados foi utilizado como instrumentos de pesquisa, uma entrevista com questionário semi-estruturado, observação de campo e análise de documentos. O estudo foi realizado nos departamentos desportivos de quatro universidades portuguesas. Através dos resultados obtidos, verificamos que as universidades tem como principal estratégia divulgar o nome da instituição e como principal objectivo, proporcionar o desporto para toda a comunidade académica.

Palavras-chave: desporto universitário, serviço desportivo, estratégias, objectivos, planeamento

\begin{abstract}
Strategies of college sports: a case study about sports in Portuguese colleges

The main focus of this study is to deepen the knowledge about college sports in Portuguese Universities, as well as to verify and understand the strategies used, the strategy planning, the objectives and the organizational structure of sport services. For this research, it was used the qualitative paradigm in case study, being it an interpretative, descriptive and phenomenological methodology. For the collection of data, it was used, as tools of research, an interview with a semi-structured questionnaire, field observation and document analysis. The study was performed in the sport departments of four Portuguese colleges. Through the results obtained, we concluded that the universities have, as their main strategy, to advertise the name of the institution and, as their main objective, to provide sports to the whole academic community.
\end{abstract}

Key-words: college sports, sport services, strategies, objectives, planning 


\section{INTRODUÇÃO}

Assim como todas as actividades humanas, o desporto também se transformou numa indústria que produz produtos e serviços para diversos consumidores(27). O desporto hoje é um mercado, disponibilizando diversos produtos aos consumidores, onde se relaciona o desporto, ao fitness, a recreação, ao lazer, actividades, bens, serviços, pessoas, lugares ou ideias. $\mathrm{Na}$ industria do desporto podemos identificar alguns segmentos específicos de acordo com a habilidade e conhecimento necessário na área como o desporto colegial, desporto profissional, administração de instalações, desporto comunitário, informações desportivas, jornalismo desportivo, administração de clubes desportivos, a industria do fitness, treinamento desportivo e medicina desportiva, administração de desportos aquáticos, consultoria e empreendimentos e os dois últimos segmentos que são o foco deste estudo, o desporto universitário através dos programas e serviços desportivos no campus universitário. O mesmo autor ainda enfatiza alguns factores que podem impulsionar o desporto universitário tais como, o aumento do número de actividades desportivas, o aumento de patrocinadores e mais investimentos para o desporto, aumento do marketing desportivo, aumento da competência na administração do desporto.

Verificando que o desporto universitário está inserido neste contexto de mercado, industria e organizações desportivas, possibilita-nos realizar uma investigação no desporto universitário de algumas instituições de Ensino Superior de Portugal. Com isso podemos verificar a organização dos serviços desportivos universitários, seus produtos e serviços bem como os principais objectivos e estratégias utilizadas para com o desporto, pois segundo Mintzberg e Quinn(16), o desenvolvimento de estratégias é um dos factores mais importantes para o sucesso da organização. Partindo desta premissa, pretendemos contribuir e aumentar os conhecimentos sobre as estratégias das universidades para com o desporto e tudo que a norteia como. Este estudo pretende identificar e compreender o contexto do desporto universitário, bem como as estratégias utilizadas e os principais objectivos para com o desporto de universidades com destaque no desporto universitário de Portugal e ainda verificar os serviços desportivos disponibilizados pelas instituições.
Sobretudo, a gestão das estratégias consiste nos objectivos que a organização tem para com o desporto, na disposição dos recursos, os serviços e produtos que serão fornecidos. Entretanto cabe-nos investigar, se é feito análises dos ambientes, para então definirem sobre os objectivos bem como as estratégias que serão formulados e implantadas. A gestão da estratégia visa um fim claro, uma selecção de objectivos, escolha, definição e posicionamento de serviços e um controlo de avaliação.

Após esta abordagem dos assuntos principais, surgem as questões pertinentes a pesquisa e as perguntas que nortearão este estudo tais como: Quais os objectivos das universidades com relação ao desporto? Quais as opções estratégicas usadas pelas universidades? Que tipo de gestão é utilizada pelas instituições? O desporto é competitivo ou recreativo? Quais as principais modalidades disponibilizadas aos alunos? Se há um planeamento por parte dos responsáveis pelos serviços desportivos das universidades? Com é a organização interna do deporto nas universidades? Qual o volume dos recursos? Quem são os tomadores de decisão? E qual a realidade dos serviços desportivos nas universidades pesquisadas? Ao contextualizar tais questões no intuito de esclarecer as expectativas do estudo, delineando sobretudo se as universidades utilizam a estratégia no desporto universitário e de que maneira é gerido os sistemas desportivos, principalmente quais são os objectivos das universidades com relação ao desporto, de que maneira é oferecido e disponibilizado aos seus estudantes, podemos finalmente definir a pergunta que norteia este estudo e o principal objectivo.

Quais são as estratégias utilizadas pelo desporto universitário em universidades de Portugal?

O estudo tem como objectivo principal identificar e compreender as estratégias das instituições do ensino superior pesquisadas em Portugal com relação ao desporto universitário, bem como os principais objectivos que levam as decisões estratégicas no contexto da pesquisa. Para tanto abordaremos no referencial teórico, o contexto do desporto universitário e conceitos sobre estratégias e todo seu processo. O desporto universitário surgiu no século XIX na Inglaterra e foi introduzido nas universidades com objectivo de melhor gerir o tempo livre dos estudantes das classes dominantes e ascendentes(2). Acredita- 
se ainda que o desporto universitário tenha surgido da observação do desporto como um meio de confraternização entre os povos, sendo um instrumento social entre a comunidade e ainda um factor importante para a melhoria da qualidade de vida das pessoas. Os primeiros passos oficiais do desporto universitário deram através do pai dos Jogos Olímpicos modernos, o Barão Pierry de Coubertin ainda no século XIX. Na sequência dos acontecimentos foram surgindo interesses em formar uma associação do desporto universitário. Foi então que em 1949, constituiu-se oficialmente a International University Sports Federation (FISU), como instituição máxima do desporto universitário e assim organizando a primeira Universiade na cidade italiana de Turin em 1959. O desporto universitário como qualquer actividade humana vem sofrendo inúmeras mudanças no decorrer nos anos se tornando também um óptimo negócio para instituições de ensino superior. O desporto universitário é formado por um conjunto de praticas lúdicas, desportivas e de formação, desenvolvendo-se nas universidades como uma actividade extra-curricular(13). O desporto pode ser inserido num contexto de prestação de serviço, desenvolvendo práticas desportivas tanto recreativas quanto competitivas para alunos, professores e funcionários da universidade. Dentro desta perspectiva ainda podemos dizer que tais praticas desportivas podem ser desenvolvidas como uma forma de lazer dos estudantes, como uma forma semi-profissional onde os estudantes competem pela instituição em troca de bolsa de estudos e na forma profissional com atletas profissionais competindo representando o nome da universidade em diversos campeonatos, tanto universitários como federados ou em ligas profissionais.

Seguindo ainda a mesma linha de raciocínio, podemos verificar que inúmeras universidades desenvolvem o desporto universitário de forma profissional, como no Estados Unidos por exemplo, onde o desporto universitário serve como um salto para os atletas ingressarem no desporto profissional e no Brasil onde o desporto universitário em muitas instituições de ensino privado utilizam o desporto como uma estratégia de marketing para a divulgação de sua marca e consequentemente visando angariar mais alunos para a instituição através do desporto de competição e assim fomentando os negócios da universidade(27).
Na realidade de hoje, as mudanças que vão surgindo a cada dia no âmbito económico e social proporciona para as organizações novos rumos e desafios a serem enfrentados. Com isso, torna-se um constante o desafio para a gestão das organizações desportivas, principalmente no desporto universitário pois lidamos com pessoas que tem seus próprios objectivos de vida e ideais a seguirem. Este ambiente é muito complexo e põem a gestão do desporto universitário e seus agentes em exigentes tarefas no intuito de obtenção de resultados e total eficiência e eficácia nos serviços prestados pelos mesmos.

Para tanto, temos consciência de que o desporto universitário pode ser melhor aproveitado através de projectos, objectivos especificamente definidos para o desporto, uma gestão profissional e ainda a utilização de estratégias na perspectiva de adquirir a vantagem competitiva. Como em qualquer actividade é necessário a profissionalização do desporto nas universidades, ter um vínculo forte entre o desporto e a instituição alinhando objectivos e formulando estratégias em conjunto no intuito de atingir a eficiência e eficácia(27).

Entretanto com as constantes mudanças no ambiente externo, exigem que as organizações desportivas estejam capacitadas para tais mudanças bem como capacitada para agir estrategicamente( ${ }^{(6)}$. O desenvolvimento de estratégias é um dos principais factores para o sucesso de qualquer organização(16).

A formulação de estratégias pode garantir que a organização alcance seus objectivos e com isso atinja a eficiência e a eficácia melhorando a performance da organização. No contexto desportivo há inúmeras organizações públicas e privadas que actuam de acordo com seus desejos, possibilidades e circunstâncias, produzindo serviços para públicos específicos, onde o desenvolvimento de estratégias é um factor importante para o sucesso das organizações ${ }^{(8)}$.

Se tratando de entidades desportivas, as organizações não tem tradição na formulação de estratégias, pois não visam a obtenção de lucro e sim na manutenção das actividades desportivas e ai podemos destacar especificamente o desporto universitário nas instituições públicas pois não visam lucros ${ }^{(9)}$.

A todo momento surgem novas tecnologias, novos produtos e serviços. As organizações necessitam de uma constante percepção frente a este ambiente. 
Novos desafios são lançados no intuito de superar as grandes evoluções nos modelos de gestão, nos produtos e serviços, pois também os consumidores estão mais exigentes e os concorrentes por sua vez estão cada vez mais eficazes.

Hoje com a aceleração das mudanças a nível de ambiente interno como novas tecnologias, novos produtos e serviços e também nas mudanças do ambiente externo como a economia instável em que vivemos hoje, exige que a empresa esteja capacitada em antecipar a estas mudanças aproveitando novas oportunidades e assim evitando as ameaças constantes. Por isso a estratégia é de suma importância, é o principal passo para que a empresa possa atingir esta capacidade de manobra(6).

Segundo Toledo(27), a estratégia principal para instituições de ensino superior é a uma estratégia de marketing, onde a universidade estará totalmente engajada na captação de novos alunos, ou seja, segmentar seu público-alvo desenvolvendo uma estratégia competitiva para atingir os objectivos da instituição. Outro aspecto importante se concentra no objectivo de divulgação da marca da universidade e dos seus produtos e serviços, popularizando-se como uma empresa voltada ao desporto.

Com isso, torna-se necessário mostrar que o desporto universitário pode ser um bom negócio para a instituição e que se for feito de maneira correcta, profissionalizada, com objectivos e metas a serem cumpridos, beneficiando os alunos, divulgando os patrocinadores, investindo no marketing e assim obtendo um retorno de alguma forma para a universidade, para alunos e para o desporto.

\section{MATERIAL E MÉTODOS}

A metodologia deste estudo baseou-se no paradigma qualitativa com estudo de caso nalgumas universidades de Portugal. Na sequencia dos acontecimentos foram estipulados os instrumentos para a colecta de dados, considerando que nos estudos qualitativos são mais utilizados a observação, a entrevista, os documentos recolhidos durante a pesquisa e assim armazenando uma grande quantidade de informações pertinentes a pesquisa. Posteriormente foi utilizado a técnica de análise e interpretação os dados recolhidos. Os principais instrumentos para a colecta de dados foram o diário de campo, observação participante passiva, onde o observador não participa dos acontecimentos, apenas regista os acontecimentos no momento em que ocorrem. O diário de campo, como colecta de dados, pode ser feito de várias formas, como memorial descritivo, no qual caracteriza-se como, segundo Molina(17) uma descrição com muitos pormenores de uma realidade vivida e se referindo aos fenómenos da consciência, as formas de pensar do indivíduo diante de situações vivenciadas. As anotações no campo da pesquisa, é uma técnica valiosa de abordagens de dados qualitativos, sendo complemento de informações obtidas por outras técnicas. A entrevista utilizada e a que mais se adequava ao estudo foi a semi-estruturada, pois o instrumento de colecta obtém informações de questões concretas, previamente definidas pelo pesquisador e que permitem realizar explorações não previstas. Molina(17) relata que, quando fazemos o uso de entrevistas semi-estruturadas, visamos garantir uma gama de informações importantes ao estudo e dando maior flexibilidade à entrevista, proporcionando mais liberdade ao entrevistado para expor seus aspectos, que pode ser relevante em se tratando de determinada temática.

Outro recurso de grande vantagem utilizado pelo pesquisador é a selecção e utilização de documentos adquiridos durante o processo de pesquisa de campo, onde podemos seleccionar revistas, jornais, artigos, documentos referentes a organização disponibilizados pelo inquerido, sendo uma fonte poderosa, de onde podem ser retiradas informações, afirmações e declarações.

No intuito de explorar ao máxima foco de estudo utilizamos a técnica de triangulação dos dados, que para Triviños (28), tem por objectivo abranger ao máximo a amplitude na descrição, explicação, e compreensão do estudo, optando por triangular o referencial teórico, as entrevistas, os documentos e a reflexão do investigador, pois o investigador não pode confiar em uma única fonte de dados. Numa perspectiva mais específica em expor o problema do estudo, tendo como definição de pesquisa, que segundo Gil(12) é um processo formal e sistemático de desenvolvimento do método científico, no intuito de descobrir as respostas para os problemas empregando procedimentos científicos. Com isso, podemos investigar que tipos de estratégias são utili- 
zadas, bem como seus principais objectivos e a missão dos departamentos desportivos das universidades, os serviços desportivos desenvolvidos nas instituições, se é feito um planeamento estratégico, qual a natureza das decisões, que recursos humanos e financeiros é dependente, quais competições e modalidades disputadas bem como o tipo de gestão utilizada pelo sistema desportivo nas universidades. Portanto, no intuito de responder tais problemas do estudo, a pesquisa é concentrada no sistema desportivo ou departamento desportivo controlador do desporto dentro da universidade e nos actores controladores deste sistema, ou seja, nas pessoas encarregadas do desporto dentro das instituições pesquisadas, bem como no descobrimento das estratégias e objectivos das universidades investigadas. Para tanto estabelecemos a pesquisa descritiva que de acordo com Gil(12), tem como objectivo e característica descrever determinada população, fenómeno ou estabelecer relação entre variáveis.

O presente estudo foi desenvolvido no contexto do Desporto Universitário em Portugal, sendo o universo investigado de quatro universidades, escolhidas através de uma análise de informações juntamente com a Federação Académica de Desporto

Universitário (FADU) com relação aos serviços desportivos desenvolvidos nas instituições bem como sua importância a nível do desporto universitário no âmbito nacional. As universidades escolhidas foram a Universidade do Minho (UM), a Universidades de Aveiro (UA), a Universidade de Lisboa (UL) e a Universidade Fernando Pessoa (UFP), caracterizando três universidades públicas e uma privada. O passo seguinte, na busca incessante do entendimento do fenómeno, foi feito a transcrição das entrevistas e a separação dos documentos arrecadados pertinentes a esta fase da investigação. A seguir utilizamos a triangulação dos dados que consiste em reunir as informações colectadas, juntamente com o referencial teórico e a reflexão do investigador para assim realizar as interpretações chegando a conclusão do presente estudo.

\section{RESULTADOS}

Nesta fase do estudo, relatamos o quotidiano das universidades pesquisadas, procurando evidenciar as principais estratégias dos serviços desportivos destas instituições de ensino superior em Portugal e também esclarecer o processo de gestão, bem como os principais objectivos e missão dos departamentos responsáveis pelo desporto universitário. Ainda dentro desta análise, destacamos também assuntos relacionados como recursos humanos e financeiros como principais investimentos por parte das universidades e com isso procurando identificar as questões acima abordadas, tendo como campo de investigação, todo o universo do desporto universitário nestas instituições que estão entre as mais importantes no âmbito do desporto universitário nacional. A análise dos dados recolhidos na investigação teve como contexto pesquisado o serviço desportivo universitário nas Universidades do Minho (UM), Universidade de Aveiro (UA), Universidade de Lisboa (UL) e Universidade Fernando Pessoa (UFP). No contexto do desporto universitário em Portugal, trata-se de três universidade públicas de renome nacional, sendo duas instituições novas e uma que é a mais antiga do país. A quarta instituição é uma universidade privada e nova de acordo com os parâmetros de universidades nacionais, mas sendo também de destaque no âmbito desportivo nacional. No processo a seguir, é feito uma análise em conjunto do material investigado. Com isso, pretende-se chegar o mais perto possível da resolução dos problemas em questão neste estudo, para na sequência chegar à conclusão.

Verificamos que nas universidades investigadas, o desporto universitário é desenvolvido e apoiado pela alta gestão das instituições com objectivo especifico de proporcionar actividades desportivas para os alunos, onde na UM e UL também conta com funcionários e a comunidade circundante a universidade como utentes do desporto. Ainda se tratando de objectivos, podemos dizer que as universidades investem no desenvolvimento do desporto visando o reconhecimento da instituição em diversos contextos através do desporto universitário.

Nos serviços desportivos encontramos discrepância entre as universidades. Tais diferenças são relacionadas no que diz respeito a instalações tanto dos departamentos desportivos quanto nas instalações desportivas para o desenvolvimento das actividades. As actividades proporcionadas aos utentes são na vertente recreativa e competitiva nas quatro institui- 
ções, havendo uma semelhança das actividades na UM e UL, onde também contam com uma gama variada de actividades. Destacamos que na UA o maior apoio é dado ao desporto competitivo e com isso as modalidades recreativas ficam desprovidas de maiores desenvolvimentos e na UFP, onde a instituição não possui instalações desportivas próprias onde sofre um decréscimo nas possibilidades de ampliar os serviços desportivos.

Os serviços desportivos nas instituições tem como principais objectivos proporcionar e organizar actividades desportivas, contribuir para a melhor qualidade de vida e ocupar o tempo livre dos alunos numa questão de lazer e convívio social. Verificamos que na UM, UA e UL os utentes dos serviços desportivos pagam taxas para usufruir das actividades, onde possuem diferentes tabelas de preços dentre os mais variados serviços disponibilizados. Entretanto na UFP os alunos não pagam qualquer tipo de taxa para usufruírem das actividades desportivas.

Os alunos ainda têm a possibilidade de representar a instituição em competições desportivas universitárias a nível regional e nacional. Destacamos a UM e a UFP que possuem equipas que participam de ligas de desporto federado. Outro factor relevante e que é comum entre as instituições investigadas, é o facto de nas competições os alunos não pagam qualquer tipo de valor para representar a universidade.

Como principal estratégia, verificamos que a estratégia de marketing é a utilizada pelos serviços desportivos, sendo totalmente voltada para divulgação da marca da instituição e o reconhecimento da universidade através do desporto universitário. Não foi detectado nenhuma intenção em fomentar os negócios da universidade através do desporto, por se tratarem de universidades públicas e o mesmo na a UFP que é uma universidade privada. As universidades apenas visam o reconhecimento nos âmbitos regional, nacional e ate internacional e a divulgação da marca e do nome da instituição.

Com relação a organização dos departamentos desportivos, verificamos alguma discrepância entre as universidades investigadas. A UM e a UL divulgam em documentos escritos a missão, visão e os objectivos do departamento desportivo, bem como divulga indicadores dos serviços desportivos. Também são elaborados documentos referentes ao planeamento anual de actividades e o planeamento das estratégias definidas pela gestão responsável do desporto. Previamente a este processo as duas instituições realizam análises internas e externas no intuito de estudar os ambientes para então delimitarem suas directrizes.

Já na UA e UFP verificamos que não há definição de missão e objectivos em documentos específicos, são apenas conjecturas internas dos gestores dos departamentos. Também verificamos a não elaboração em documentos sobre o planeamentos estratégicos específicos para o desporto. Apenas são elaborados documentos referentes a planeamento anual de actividades, projectos e orçamentos anuais.

Tais factores anteriores são diagnosticados devido ao tipo de gestão por parte dos serviços desportivos destas instituições. Verificamos que na UM a gestão está a cargo de aproximadamente 10 profissionais que conta com uma equipa de trabalho entre instrutores e colaboradores que corresponde a amplitude do desporto naquela instituição, o mesmo ocorrendo com a UL que possui uma equipa de 6 profissionais e mais instrutores e colaboradores suficiente para o desenvolvimentos das funções organizacionais. Já na UA, notamos uma carência na parte da gestão onde apenas dois gestores profissionais comandam o desporto não suprindo as necessidades reais do desporto na UA, contando com instrutores responsáveis pelas modalidades desportivas. Entretanto na UFP a gestão é feita apenas por uma pessoa que foi denominada semi-profissional pois está em regime parttime contando com a colaboração do presidente da associação responsável pelo desporto em carácter voluntario e com os técnicos das equipas.

Entretanto, esta gestão do deporto universitário, depende muito dos recursos financeiros para a sua existência. Na UM 60\% dos recursos são suportados pelo Serviço de Acção Social e os outros $40 \%$ é relativo as taxas pagas pelos utentes dos serviços desportivos. A UA a taxa dos alunos suporta $50 \%$ do orçamento sendo os outros $50 \%$ suportado pelo Serviço de Acção Social e pela Câmara de Aveiro. Na UL não forma fornecidos indicadores de percentagem apenas foi relatado que o orçamento é suportado pela Reitora, pelo Serviço de Acção Social e pela taxa dos utentes dos serviços desportivos. Já na UFP o orçamento para o desporto é totalmente suportado pela Reitoria da instituição. 


\section{DISCUSSÃO}

O estudo em conclusão passou pelo universo do desporto universitário de instituições de renome em Portugal, tendo como proposta investigar as principais estratégias e objectivos dos serviços desportivos nas universidades pesquisadas. Para tanto realizamos a pesquisa baseado no paradigma qualitativo que tem como metodologia interpretativa fenomenológica e descritiva no qual interpreta as acções humanas. Com isso a investigação partiu de inquéritos com os responsáveis dos serviços desportivos das universidades do Minho, Lisboa, Aveiro e Fernando Pessoa, com objectivo do descobrimento sobre o contexto do estudo para posteriormente responder a pergunta as questões que envolvem a investigação.

As universidades pesquisadas apoiam o desenvolvimento das práticas desportivas através dos departamentos desportivos responsáveis pelo serviço desportivo universitário. Os principais objectivos para o desenvolvimento do desporto por parte da instituição são o visionamento e reconhecimento da universidade através do desporto. Este reconhecimento pode ser através do desporto competitivo que participa em campeonatos universitários em ambas as instituições e no desporto federado com a UA e UFP. Este reconhecimento também pode vir através do desporto recreativo como na UM e UL onde os serviços desportivos são estendidos também a funcionários e população em geral circundante a universidade. Para tanto o estabelecimento de estratégias por parte das universidades gira em torno do marketing da universidade, com objectivo de divulgar o nome e a marca da instituição. As universidades do Minho e de Lisboa ainda estabelecem uma estratégia de diversificação dos seus serviços, propiciando uma variada gama actividades tendo cada universidade a sua particularidade.

Com relação a estrutura das organizações desportivas, verificamos as universidades do Minho e de Lisboa se diferem da UA e UFP, no estabelecimento de estratégias, nos planeamentos, nas tomadas de decisões e nas elaborações dos documentos contando uma missão, visão e objectivos para com o desporto. Tais diferenças podem ser justificadas pelos diferentes apoios dado aos serviços desportivos por parte da alta gestão das instituições inqueridas. Verificamos que as gestões dos serviços desportivos são de capacidades diferenciadas para o desenvolvimento do desporto universitário nas instituições, pois como pudemos verificar através dos dados recolhidos a UA e UFP contam com um números de pessoas na gestão do desporto bem reduzido comparado a UM e UL.

Entretanto, não podemos deixar de destacar o empenho por parte destes gestores da UA e UFP que lutam constantemente para o desenvolvimentos do desporto nas instituições apesar de suas dificuldades, principalmente por parte da UFP que não possui instalações desportivas próprias, mas que relatou está em andamento um projecto para a construção de um pavilhão desportivo. Outro factor importante e relevante para este estudo é que nenhuma das universidades investigadas possui o curso de Educação Física o que seria uma mais valia com relação a recursos humanos e recursos de instalações desportivas. Finalizando este estudo, face a necessidade de elucidar a importância do estudo das estratégias bem como sua utilização por parte dos serviços desportivos universitários no objectivo de maximizar o desenvolvimento do desporto universitário em todos os âmbitos. Esperamos que este estudo contribua para um maior conhecimento do contexto desportivo universitário, bem como dos serviços desportivos, pois temos consciência que o universo pesquisado é muito maior, parecendo-nos importante o aumento de estudos nesta área que no nosso entender é carente de referências bibliográficas.

\section{CORRESPONDÊNCIA}

Carlos Jorge Pinheiro Colaço

Faculdade de Motricidade Humana

1495-688 Cruz Quebrada Lisboa Portugal

E-mail: ccolaco@utl.fmh.pt 


\section{REFERÊNCIAS}

1. Ackoff RL (1978). Planejamento Estratégico. Rio de Janeiro: Livros Técnicos e Científicos.

2. Alegre CA (1988). Desporto Universitário em Portugal. Horizonte 4, 24: 209 - 212.

3. Barroso ML, Araújo AG, Keulen GE, Braga, RK Krebs, RJ (2007). Motivos de Pratica de Esportes Coletivos Universitários em Santa Catarina. $6^{\circ}$ Fórum Internacional de Esportes, Florianopolis - SC.

4. Almeida MI (2001). Manual do Planejamento Estratégico: desenvolvimento de um plano estratégico com a utilização de planilhas excel. São Paulo: Editora Atlas.

5. Campos AP (2000). O Planejamento Estratégico e as Competências de Perrenoud para o Oficio Docente: considerações para a Educação Física. Revista Brasileira Ciências do Esporte 23, 2: 149 - 163.

6. Chiavenato I (1994). Administração, teoria, processo e prática. São Paulo: Makrom Books.

7. Correia A (1997). Estratégias das Federações Portuguesas, Horizonte 13,77: 20

8. Correia A, Pires G, Colaço C (2000). Estratégias das Principais Federações Portuguesas no Ciclo Olímpico de Atlanta (1993 a 1996). Horizonte 16, 93: 24-30.

9. Correia A (1994). Estratégias nas Federações: emergência de uma necessidade. Ludens 14, 2: $19-26$.

10. Farias A, Freitas L, Santos P (2006). Implementação de Estratégias: estudo de caso em uma instituição pública. Revista ANGRAD 7, 3: 73 - 93.

11. Freire A (1998). Estratégia, sucesso em Portugal. Lisboa: Editora Verbo.

12. Gil A (1991). Como Elaborar Projetos de Pesquisa. São Paulo: Atlas.

13. Kay P (1999). Desporto no Ensino Superior. Ludens 15, 1/2: $60-64$.

14. Lima A, Veloso M, Pereira R (2000). Reconhecer o Desporto do Ensino Superior de Lisboa. Horizonte 14, 94 : $20-25$.

15. Lüdke M, André M (1986). Pesquisa em Educação: Abordagens Qualitativas. São Paulo: EPU.

16. Mintzberg H, Quinn J (2001). O Processo da Estratégia. Porto Alegre: Bookman.

17. Molina N (2001). Pesquisa Qualitativa na Educação Física: Alternativas Metodológicas. Porto Alegre: Sulina.

18. Oliveira D (1997). Planejamento Estratégico: conceitos, metodologia e prática. São Paulo: Atlas.

19. Oliveira L, Picinatto A, Vieira W (2005). Associativismo Empresarial: um estudo de caso da implementação do pla- nejamento estratégico participativo em uma rede de materiais de construção. Revista ANGRAD 6, 4: 65 - 84 .

20. Pires G (2005). Gestão do Desporto: desenvolvimento organizacional. Porto: APOGESD.

21. Pires G, Lopes JP (2001). Conceito de Gestão do Desporto; novos desafios, diferentes soluções. Revista Portuguesa de Ciência do Desporto 1, 1: 88 - 103.

22. Saccol AI (2005). Alinhamento Estratégico da Utilização da Internet e do Comercio Electrónico: os casos Magazine Luiza e Fleury. RAC 9, 2: $59-80$.

23. Scola R (2004). Competências organizacionais alinhadas à estratégia e aos processos internos. Global Manager 4, 6: 109 - 125.

24. Soares J, Correia A (2005). A Tomada de Decisão Estratégica e a Estrutura Organizacional nas Associações Desportivas de Modalidade. Revista Portuguesa de Gestão do Desporto 2, 1: $21-30$.

25. Tauhata TL (2004). Redes e Alianças Estratégicas. ERAelectrônica 3, 1: 1 - 25.

26. Toaldo AM, Luce FB (2006). Estratégia de Marketing: contribuições para a teoria em marketing. RAE- electrónica,46, 4: $25-35$.

27. Toledo R (2006). Gestão do Esporte Universitário: uma importante estratégia de marketing para as universidades. São Paulo: Aleph.

28. Triviños AN (1987). Introdução à Pesquisa em Ciências Sociais: A Pesquisa Qualitativa em Educação. São Paulo: Atlas.

29. Zilber MA, Piekny EE (2005). Estratégias de Fusões e Aquisições. RAC 9, 3: 169 - 191.

\section{Sítios da Web Visitados}

Associação Académica da Universidade de Aveiro: www.aauav.pt Associação Cultural e Desportiva da Universidade Fernando Pessoa: $w w w . a c d . u f p . p t$

Desporto da Universidade de Lisboa: www.desporto.ul.pt European University Sports Association: www.eusa.eu Federação Académica do Desporto Universitário: www.fadu.pt International University Sports Federation: www.fisu.net Serviços de Acção Social da Universidade do Minho: www.sas.minho.pt

Serviço de Acção Social da Universidade de Aveiro: www.sas.ua.pt

Serviço de Acção Social da Universidade de Lisboa: www.sas.ul.pt

Universidade de Aveiro: www.ua.pt

Universidade de Lisboa: $w w w . u l . p t$

Universidade Fernando Pessoa: www.ufp.pt

Universidade do Minho: www.uminho.pt 